\title{
Anatomical and Microsurgical Implications in Total and Midarm Transplantation
}

\author{
Martin Iglesias, MD ${ }^{1}$ Fernanda Salazar-Hernández, MD ${ }^{1}$ María F. Ramírez-Berumen, MD ${ }^{1}$ \\ Patricia Butrón, $M^{1}$ Josefina Alberú-Gómez, $M^{2}$ Rafael P. Leal-Villalpando, $M^{3}$ \\ Jorge Zamudio-Bautista, MD $^{3}$ Victor Acosta, MD $^{3}$ Luis A. Jauregui-Flores, MD \\ Verónica Espinosa-Cruz, $\mathrm{MD}^{5}$ Jorge Vázquez-Lamadrid, $\mathrm{MD}^{5}$ Judith González-Sánchez, $\mathrm{MD}^{6}$ \\ Carlos A. Hinojosa, $M^{7}$ Hugo Laparra-Escareño, MD $^{7}$ Juan Montejo-Vargas, MD $^{8}$ \\ Julio Macias-Gallardo, MD ${ }^{9}$
}

${ }^{1}$ Department of Plastic Surgery, Instituto Nacional de Ciencias Médicas y Nutrición "Salvador Zubirán," México City, México

2 Department of Transplants, Instituto Nacional de Ciencias Médicas y

Nutrición "Salvador Zubirán," México City, México

${ }^{3}$ Department of Anesthesiology, Instituto Nacional de Ciencias

Médicas y Nutrición "Salvador Zubirán," México City, México

${ }^{4}$ Department of Anesthesiology, Hospital General Gea Gonzalez,

Secretaria de Salud, México

${ }^{5}$ Department of Radiology and Imaging, Instituto Nacional de Ciencias

Médicas y Nutrición "Salvador Zubirán," México City, México

${ }^{6}$ Department of Psychiatry, Instituto Nacional de Ciencias Médicas y

Nutrición "Salvador Zubirán," México City, México

${ }^{7}$ Department of Vascular Surgery, Instituto Nacional de Ciencias

Médicas y Nutrición “Salvador Zubirán," México City, México

8 Department of Orthopedic, Instituto Nacional de Ciencias Médicas y

Nutrición "Salvador Zubirán," México City, México

${ }^{9}$ Laboratory of Clinical Neurophysiology, Instituto Nacional de Ciencias Médicas y Nutrición "Salvador Zubirán," México City,

México

J Reconstr Microsurg Open 2017;2:e94-e102.
Address for correspondence Martin Iglesias, MD, Department of Plastic Surgery, Instituto Nacional de Ciencias Médicas y Nutrición “Salvador Zubirán," Monte de Antisana 47, Jardines en la Montaña, Tlalpan, Mexico City 14210, México

(e-mail: iglesias@drmartiniglesias.com).

\begin{abstract}
\section{Keywords}

- arm transplantation

- vascularized composite allotransplantation

- hand transplantation
\end{abstract}

Background Arm transplantations are performed less frequently than forearm and hand transplantations. We present the surgical and microsurgical technique and its relationship with the clinical results in a patient with bilateral arm transplantation. Methods A 51-year-old male patient underwent bilateral arm transplantation in October 2015. The right arm was transplanted at the glenohumeral joint. The vascular repair was at the axillary level, and the nerves were repaired at their origin. The total ischemia time was 3 hours and 48 minutes. The left arm was transplanted at the midhumeral level; all muscles were completely transplanted. The nerves were repaired at the distal third of the arm. Additionally, terminolateral neurorrhaphy was performed from the donor musculocutaneous nerve to the recipient radial nerve. The total ischemia time was 6 hours and 35 minutes.

Results At 15 months posttransplantation, the right shoulder had an abduction of 90 degrees and muscle strength of M4; flexion of 100 degrees and M4; internal and external rotation of M1; elbow flexion of 120 degrees and M3; elbow extension of M5; received

May 15, 2017

accepted after revision

June 11, 2017
DOI https://doi.org/

$10.1055 / \mathrm{s}-0037-1604342$.

ISSN $2377-0813$
Copyright $\odot 2017$ by Thieme Medical

Publishers, Inc., 333 Seventh Avenue,

New York, NY 10001, USA

Tel: +1(212) 584-4662.
License terms

(이) $\Theta \circledast$ 
pronosupination of $\mathrm{M} 2$; and wrist extension of $\mathrm{M} 2$. There was no mobility in the fingers. The left transplanted limb had total elbow flexion and extension of M5, pronosupination of M2, wrist extension of M4, and finger flexion of M2. Both extremities had thermal sensitivity that allowed discrimination of cold and heat with residual deep pressure.

Conclusion Although the functional results of arm transplantation are so far unknown, they may be considered beneficial compared with the devastating disability caused by arm amputation.

Arm transplantations are performed less frequently than forearm and hand transplantations. One of the main reasons for this is the great distance of nerve regeneration required to reinnervate a large number of muscles, mainly the intrinsic musculature of the hand. ${ }^{1-3}$ Therefore, the patient requires intense and prolonged rehabilitation, as well as a greater multidisciplinary support to obtain basic function of the limb. Another factor that limits arm transplantation is the large amount of muscle mass present in the transplant, which demands shorter periods of ischemia and carries the risk of greater complications, including death. ${ }^{4,5}$

To the best of our knowledge, only eight arm transplants have been performed in seven patients to date. Of these, one was at the level of the proximal third of the humerus, five were transhumeral, and two were at the distal third of the humerus. Five patients were reported in the medical literature, and the last two were reported in electronic media and social networks. ${ }^{1,2,6-13}$ Functionally, elbow flexion and extension was satisfactory and recovered quickly. Flexion and extension of the wrist and fingers was also obtained. However, function of the intrinsic musculature was not reported. The reported sensitivity was of a protective type with a distinction between thermal stimuli., ${ }^{1,8,9,14}$

The most proximal transplantation to date was performed in Germany by Höhnke et al. ${ }^{6}$ The transplant was distal to the insertion of the deltoid muscle and had only one functional report with a follow-up of 2 years. ${ }^{14}$

In Mexico, an upper limb transplantation protocol was implemented in 2007. After performing our first successful case of bilateral forearm transplantation in $2012,{ }^{15}$ we performed an evaluation of 131 patients, with amputation of a segment of the upper limb, in whom hand transplantation was considered a treatment option. Most of these cases were arm amputations at varying levels. ${ }^{16}$

The final functional results of arm transplantation are so far unknown, mainly regarding the reinnervation of intrinsic muscle; ${ }^{9,14}$ however, due to the devastating disability that arm amputation causes, it is considered beneficial to perform a bilateral arm transplantation in an ideal patient. ${ }^{7}$ In the present case, one arm was transplanted at the level of the glenohumeral joint, and the other at the level of the middle third of the humerus. As arm transplantation is very rare, extensive knowledge of the upper limb anatomy is not common in any specialty. ${ }^{17}$ Therefore, the aim of this article was to elucidate the surgical, microsurgical, and anatomical considerations that enabled this bilateral arm transplantation.

\section{Methods}

A 51-year-old male patient suffered a high-voltage electric burn in January 2012 with an entry point on the upper extremities and an anterior thorax exit point. This led to the amputation of the upper right extremity at the level of the proximal epiphysis of the humerus and amputation of the left arm at the transhumeral level. The patient was unable to adapt to mechanical prostheses. His Disabilities of the Arm, Shoulder and Hand (DASH) score was 75.83 points. The patient was healthy without psychiatric history, personality disorders, anxious or narcissistic traits, or depressive or anxious behavior secondary to traumatic episodes. He also had optimal support networks and they understood the risks and consequences of the procedure. Hence, this patient was considered an ideal candidate for upper limb transplantation.

The right stump had a stable cutaneous cover with an osteoporotic humeral head. There was atrophy of the deltoid, supraspinatus, infraspinatus, and latissimus dorsi muscles. The upper half of the pectoralis major was preserved. There was a palpable axillary artery and positive Tinel's sign at the infraclavicular level (-Fig. 1).

The left stump was at the level of the junction of the middle and distal thirds of the humerus with a stable skin cover. The shoulder muscles were functional. There was partial conservation of the origin of the two heads of the biceps brachii muscle and the lateral and long heads of the triceps brachii muscle, as well as the upper portion of the pectoralis major. The coracobrachialis muscle was healthy. There was a positive Tinel's sign $5 \mathrm{~cm}$ proximal to the stump. The brachial artery was palpable at the upper third of the stump (-Fig. 1).

Bilateral upper limb transplantation was performed in October 2015. The right limb was transplanted at the glenohumeral joint and the left limb at the middle third of the arm. The multiorgan donor was a 33-year-old man with brain death. The human leukocyte antigen (HLA) donor/recipient mismatch was five of six, with a reactive panel of antibodies against major histocompatibility complex class I ( $2 \%$ ) and class II (7\%) and negative cross tests. The ABO and Rh groups of both donor and recipient were A positive. Serology of donor/ 


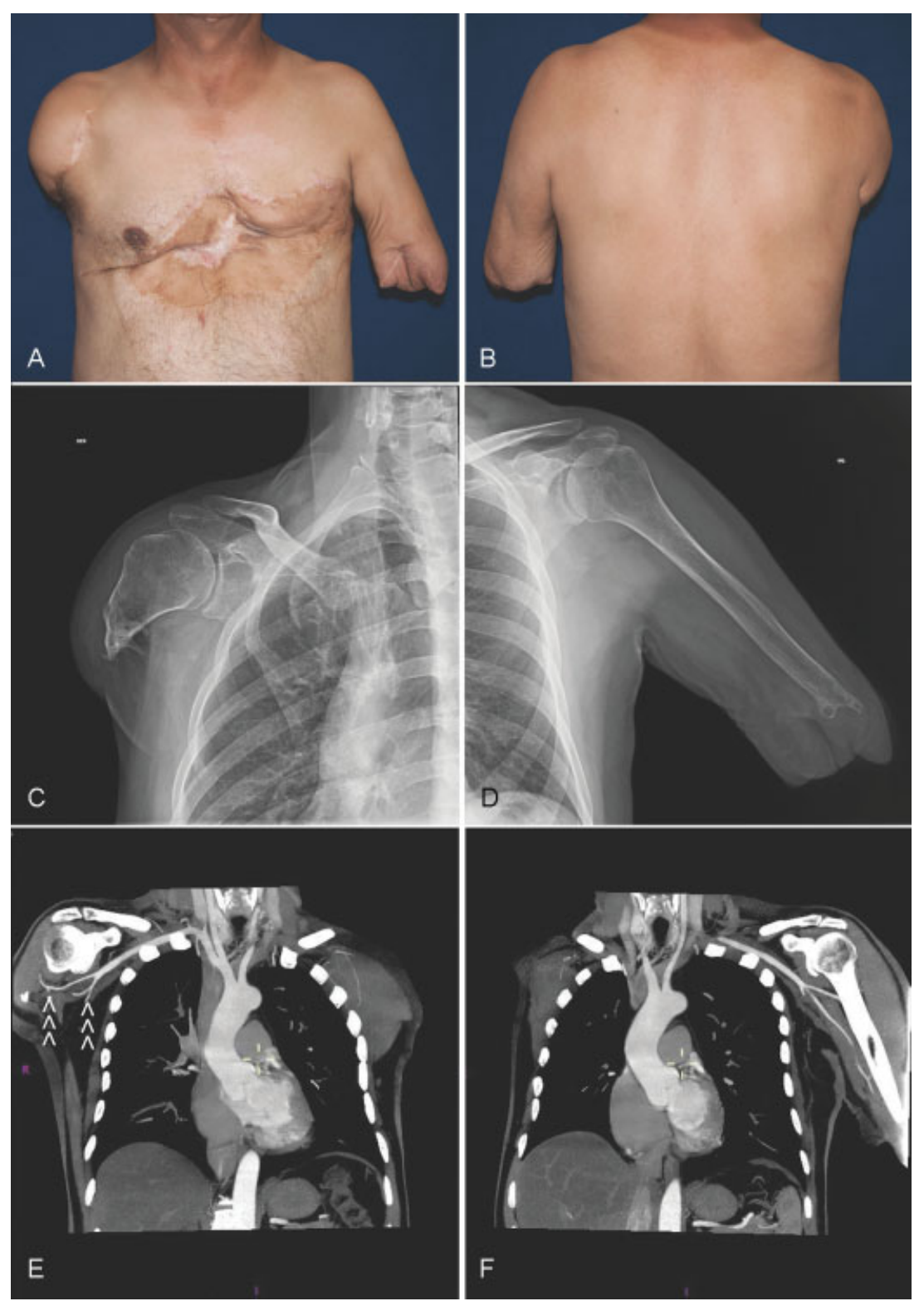

Fig. 1 Preoperative status. (A) Extensive scarring caused by exit point of energy, atrophy of the right pectoralis major muscle, and redundancy of skin cover of the left stump. (B) Atrophy of the right supraspinatus, infraspinatus, and latissimus dorsi muscles. (C) Remnant proximal epiphysis of the humerus with significant osteopenia and integrity of the glenohumeral joint. (D) Important osteopenia of the distal third of the remaining humerus compared with the proximal third. (E) The axillary artery is visualized until its third anatomical portion with presence of the anterior and posterior humeral circumflex arteries (arrows). (F) Brachial artery in good condition in the proximal third of the left arm.

recipient was: cytomegalovirus $-/+$; rubella $+\mid+$; Toxoplasma $-/-$; and Epstein-Barr virus $+/+$. The donor and recipient were in separate hospitals 1 hour apart.

\section{Recovery}

Two surgical teams recovered the upper extremities simultaneously with the recovery of the solid organs. Tourniquet control was not used.

\section{Right arm}

The deltoid muscle was disassembled from the clavicle. The axillary artery and vein were identified and dissected distal to the subclavian artery and vein. The tendons of the pectoralis major, latissimus dorsi, and teres major were identified and cut $3 \mathrm{~cm}$ from their insertion on the humerus. Disinsertion of the origin of the long portion of the triceps brachii, long and short portion of the biceps brachii, and coracobrachialis was done. The glenohumeral joint capsule was sectioned at the neck of the scapula along with the tendons of the supraspinatus, subscapularis, infraspinatus, and teres minor. The brachial plexus was identified at the lateral, posterior, and medial cords and was tagged and sectioned. The axillary artery was cannulated at the level of its junction with the subclavian artery. The axillary vein was sectioned distal to the subclavian vein. The arm was perfused with $3 \mathrm{~L}$ of histidine-tryptophanketoglutarate (HTK) solution at $4^{\circ} \mathrm{C}$ until clear drainage was obtained through the axillary vein. The recovery time was 3 hours. 


\section{Left arm}

A circumferential incision was made proximal to the insertion of the deltoid muscle. The long and lateral heads of the triceps brachii, tendons of the short and long portion of the biceps brachii, and coracobrachialis muscle were identified and released from their origins. The cephalic vein was identified and dissected as were the brachial artery and veins. Humeral osteotomy was performed distal to the insertion of the deltoid muscle. The median, radial, ulnar, and musculocutaneous nerves were identified and sectioned at their proximal thirds. The brachial artery was cannulated at its proximal third in the arm and the veins were sectioned. The arm was perfused with the same solution as the right arm until clear drainage was obtained through the veins. The recovery time was 1 hour and 57 minutes.

\section{Right Arm Implantation}

In the residual stump, an infraclavicular incision was made with extension toward the anterior border of the axilla and was continued along the previous surgical scars. The tendon of the pectoralis major muscle was removed from the humerus. The axillary artery and vein were identified and dissected from below the minor pectoralis muscle up to the subclavian artery and vein. The median, musculocutaneous, ulnar, radial, and axillary nerves were then identified and tagged. The residual tendons of the latissimus dorsi and teres major muscles were tagged and released from the humerus. The residual deltoid muscle was detached from the clavicle and the spine of the scapula. Subsequently, the joint capsule of the shoulder was sectioned from the humeral head along with the tendons of the supraspinatus, infraspinatus, teres minor, and subscapularis muscles. The tendinous origin of the long portion of the biceps brachii was preserved. The humeral head was resected, leaving the glenoid cavity and joint capsule.

The upper right extremity was removed from cold ischemia. The extremity was perfused with a buffer solution consisting of $800 \mathrm{~mL}$ of Hartmann's formula and $80 \mathrm{~g}$ of human albumin. The joint capsule of the glenoid cavity was joined to the humeral head joint capsule with interrupted sutures of Nylon number 2. The donor axillary artery was repaired end-to-end in its first anatomical portion with the third anatomical portion of the recipient axillary artery, with 6-0 Nylon suture in a continuous pattern. The arterial perfusion was immediate. The limb was allowed to bleed for 3 minutes with a blood loss of approximately $400 \mathrm{~mL}$. The axillary vein was repaired terminal-lateral in its first anatomical portion using the parachute technique with continuous 6-0 Nylon suture. The total ischemia time was 3 hours and 48 minutes ( 3 hours and 11 minutes of cold ischemia and 37 minutes of warm ischemia).

Tenorrhaphy of the long portion of the biceps brachii was performed distal to the glenohumeral joint capsule; tenorrhaphy of the supraspinatus, infraspinatus, teres minor, and subscapularis was also performed. The tendons of the latissimus dorsi, teres major, and the long portion of the triceps brachii were repaired. The short portion of the biceps brachii and the coracobrachialis muscle were reinserted into the coracoid apophysis with Nylon number 1 . Microsurgical epineural nerve repair of the axillary, musculocutaneous, radial, ulnar, and lateral and medial branches of the median nerves was performed using 9-0 Ethilon at magnification $\times 24$. Prolene number 1 was used to reinsert the pectoralis major muscle on the humerus and the deltoid muscle on the clavicle. Finally, we performed hemostasis, placement of Jackson-Pratt type closed silicone drainage, and remodeling of the cutaneous flaps.

\section{Left Arm Implantation}

Incisions were made in the medial and lateral regions of the residual stump and connected with a transverse incision. An anterior and posterior cutaneous flap was dissected from distal to proximal up to the deltoid muscle insertion on the humerus. The brachial artery and veins, as well as the median, ulnar, and radial nerves, and the residual muscular masses of the biceps and triceps brachii were identified. Approximately $10 \mathrm{~cm}$ of all nerves were dissected up to the proximal third of the humerus. The recipient humerus was cut distal to the insertion of the deltoid muscle.

The left extremity was removed from cold ischemia. A distal humeral osteotomy was performed at the insertion of the deltoid. The osteosynthesis was performed with an $8.5 \times 260 \mathrm{~mm}$ Fixion intramedullary nail. One brachial vein and then the brachial artery were repaired via end-to-end anastomosis with 8-0 continuous suture. Revascularization was immediate without any complications. Total ischemia time was 6 hours and 35 minutes (5 hours and 57 minutes of cold ischemia and 38 minutes of warm ischemia).

Tenorrhaphy of the long portion of the biceps brachii and long portion of the triceps brachii was performed with the Bunnell technique. The tendons of the short head of the biceps brachii and the coracobrachialis muscle were inserted in the coracoid process. Myorrhaphy was performed of the long and lateral portions of the triceps brachii, as well as both heads of the biceps brachii and the coracobrachialis muscle to the corresponding remnant muscles using the "piggyback" technique.

End-to-end epineural nerve repair of the median, ulnar, and radial nerves was performed with 9-0 suture (-Fig. 2). Neurorrhaphies were performed $7 \mathrm{~cm}$ proximal to the elbow joint. Finally, end-lateral neurorrhaphy of the donor musculocutaneous nerve to the proximal third of the recipient radial nerve was performed.

The total blood loss was 13,000 mL. Twenty-two globular packages, 14 plasma packages, 30 cryoprecipitate packages, and 3 platelet apheresis were transfused. The total surgery time was 17 hours (-Fig. 3 ).

\section{Secondary Surgeries}

At postoperative day 12 , a seroma was detected at the right axillary level that warranted surgical evacuation. Intraoperatively, there was dehiscence of the insertion of the short portion of the biceps brachii and the coracobrachialis muscle in the coracoid process, as well as at the insertion of the pectoralis major on the humerus. Seroma evacuation and reconstruction of the abovementioned tendons were performed with fascia lata tendon allografts. The postoperative course was uneventful. 


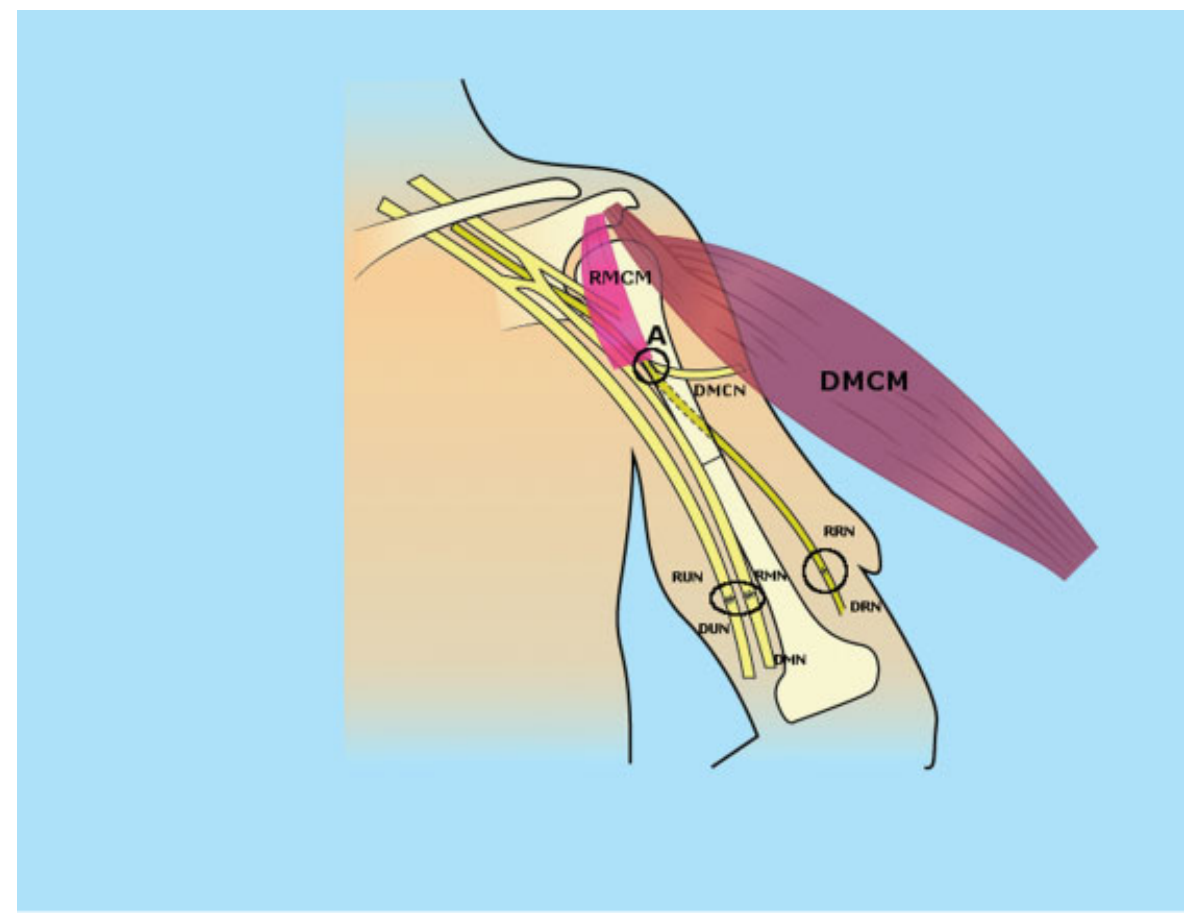

Fig. 2 Neurorrhaphies of the left arm. Circle A shows end-lateral neurorrhaphy of the donor musculocutaneous nerve to the recipient radial nerve. DMCM, donor musculocutaneous muscle; DMCN, donor musculocutaneous nerve; DMN, donor median nerve; DRN, donor radial nerve; DUN, donor ulnar nerve; RMCM, recipient musculocutaneous muscle; RMN, recipient median nerve; RRN, recipient radial nerve; RUN, recipient ulnar nerve. Black circles show the sites of neurorrhaphy.

At postoperative day 306 and according to the Medical Research Council scale for muscle strength, the patient presented as M1 in right elbow flexion and M2 in the left. Hence, bilateral transposition of the muscular remnants of the pectoralis major was performed. An Achilles tendon allograft was used to firm up their insertion in both biceps brachii tendons.

\section{Results}

The patient was healthy with a blood pressure of $131 / 87 \mathrm{~mm}$ $\mathrm{Hg}$ and was placed on a triple maintenance immunosuppression scheme (tacrolimus, mycophenolate mofetil, and prednisone) with corporal integrity. The skin color of both

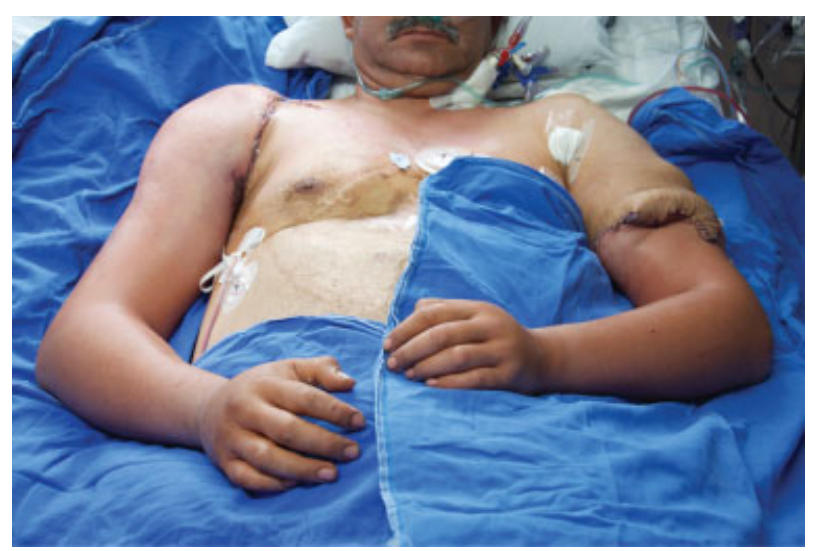

Fig. 3 Immediate postoperative. Normal coloration, tissue perfusion, and temperature. Moderate edema in both extremities with a stable patient in intensive care. extremities was similar to the recipient. The right upper extremity was 1 inch longer than the contralateral side. Arterial pulses and palpable veins were present. Temperature, capillary refill, and nail growth were normal. There was decreased hair growth without sweating.

The right shoulder had a similar shape and volume to the contralateral side. There was abduction (deltoid, supraspinatus) of 90 degrees and M4 and flexion (deltoid, coracobrachialis, and supraspinatus) of 100 degrees and M4. Internal and external rotation was M1, elbow flexion (biceps brachii, pectoralis major) was 120 degrees and M3, elbow extension was M5, pronosupination was M2, and wrist extension was M2. There was no mobility of the fingers. There was no pain during active mobility. There was thermal sensitivity allowing discrimination of cold and heat.

The left limb was transplanted with redundant skin at the level of the union with the graft. Total elbow flexion and extension was M5, pronosupination was M2, wrist extension was M4, and finger flexion was M2. There was thermal sensitivity allowing discrimination of cold and heat with residual deep pressure ( $\mathbf{- F i g s .} \mathbf{4}, \mathbf{5}$, and $\mathbf{6}$ ).

The patient regained his corporal integrity and improved his self-esteem and sense of well-being within a social group. The functions obtained so far allowed him to eat by himself, prepare food, go to the bathroom alone during night, open doors, carry bags, swim, switch on and off the lights, and play with his family.

The patient stated, "Until now, the function obtained has justified the risks involved in transplantation and immunosuppression." 

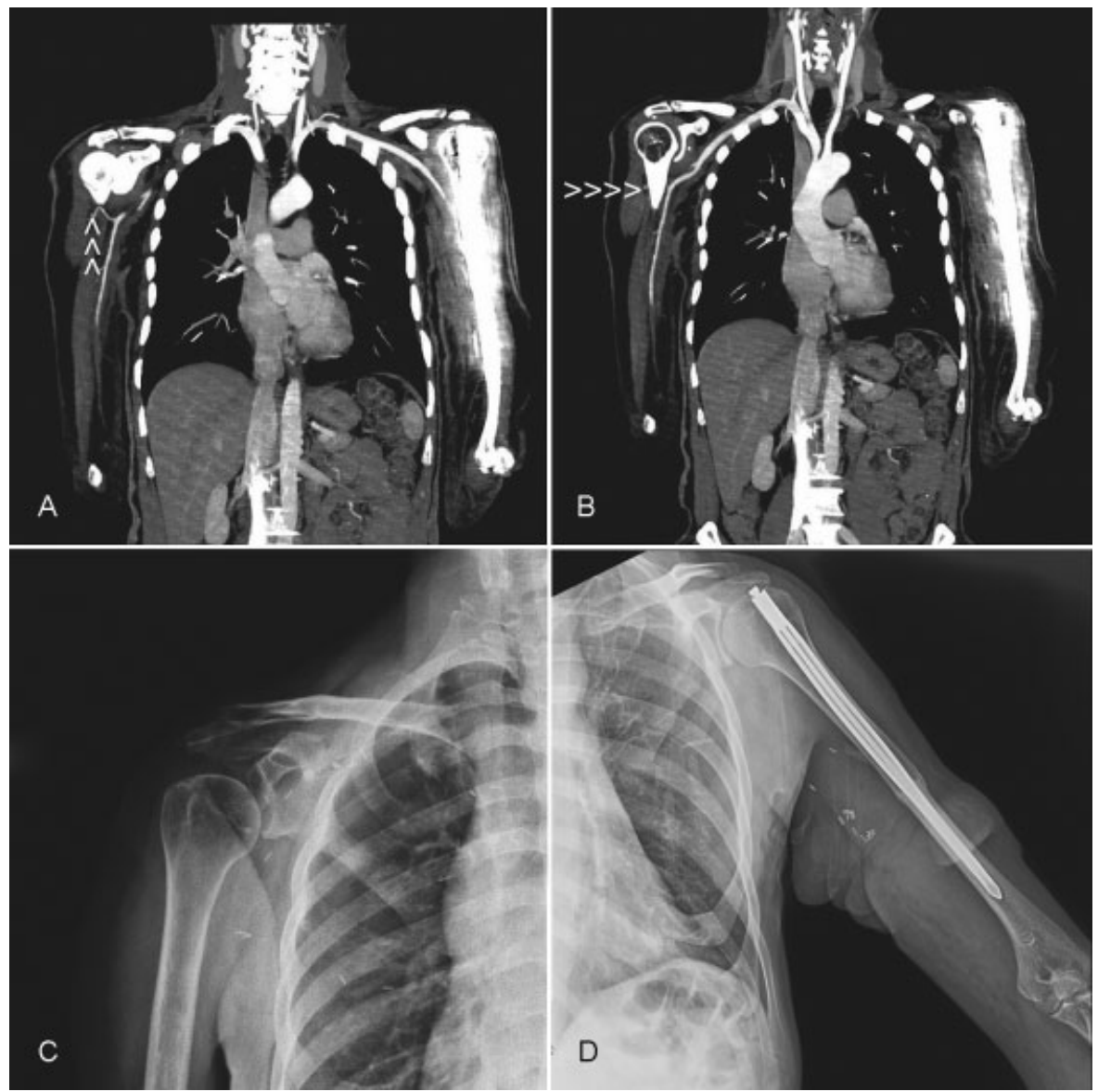

Fig. 4 Twelve postoperative months. (A) Angiotomography showing anterior circumflex humeral artery (arrows). (B) Angiotomography showing posterior circumflex humeral artery (arrows). (C) Humeral head with joint integrity and increased articular space, humeral head descended. (D) Bone consolidation of left humerus.

\section{Discussion}

The functional disability caused by transhumeral amputation is greater than the disability produced by transradial amputation. Although the overall function of a transhumeral transplant may be less than that of a distal forearm transplant, the magnitude of the potential improvement is greater due to the greater preoperative disability. ${ }^{2}$ Thus, the riskbenefit ratio favors transplantation. ${ }^{7}$

The surgical technique for hand and forearm transplantation has been extensively described; ${ }^{1,18-24}$ however, the technique for arm transplantation has been insufficiently reported, as only few cases of arm transplantation have been performed. ${ }^{1,2,6-13}$ Hence, we have described the surgical technique and some microsurgical concepts of arm transplantation at the level of glenohumeral joint and a midhumeral transplant, as well as the clinical results.

Use of tourniquet is recommended for recovering the upper extremity. This avoids hemodynamic destabilization of the donor and allows fast recovery, usually in less than an hour. $2,7,17,22,25$ However, when the limb to be recovered has a large amount of muscle mass (e.g., an arm), it is necessary to decrease the ischemia time. Hence, we do not advice the use of a tourniquet. The right arm recovery was complex, meticulous, and caused bleeding. Therefore, we planned to perform the back table procedure during the recovery time while continually maintaining the arterial circulation of the arm.

All arm muscles were recovered in their entirety, including the deltoid muscle. The right blood vessels were recovered up to the origin of the subclavian vessels to preserve the anterior and posterior circumflex humeral arteries and thus maintain the circulation of the humeral head. This caused a total recovery procurement time of 3 hours but without ischemia and without hemodynamically destabilizing the donor.

The left arm was also recovered without the use of a tourniquet, and all the muscles to be transplanted were kept intact. The time of left arm recovery was also prolonged but without ischemia time.

The total ischemia time reported in arm transplantation ranges from 1.5 to 9 hours, the most frequent being 5 to 6 hours of total ischemia. ${ }^{2,7,8,26}$ In our case, the total ischemia time in the right arm was 3 hours and 48 minutes and in the left arm was 6 hours and 35 minutes, which is in accordance with previously reported ischemia times.

Despite these prolonged ischemia times and the large amount of muscle mass involved, our patient remained stable hemodynamically and biochemically after the revascularization of each arm, as well as at the end of surgery, without symptoms of reperfusion syndrome. Reperfusion 

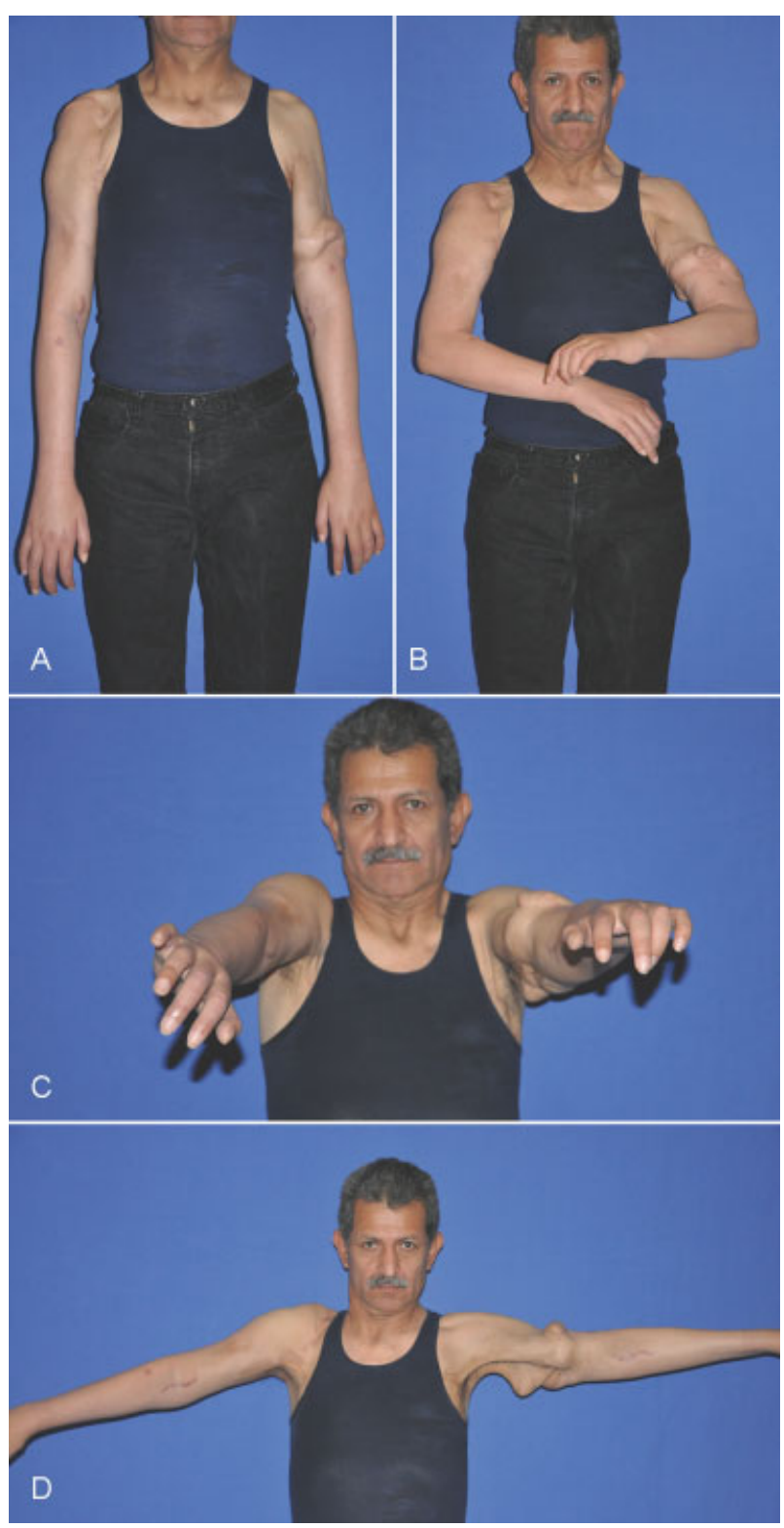

Fig. 5 Functionality at 15 months. (A) Recovered body integrity with good skin color match. (B) Elbow flexion. (C) Total flexion of right shoulder. (D) Nearly complete abduction of right shoulder.

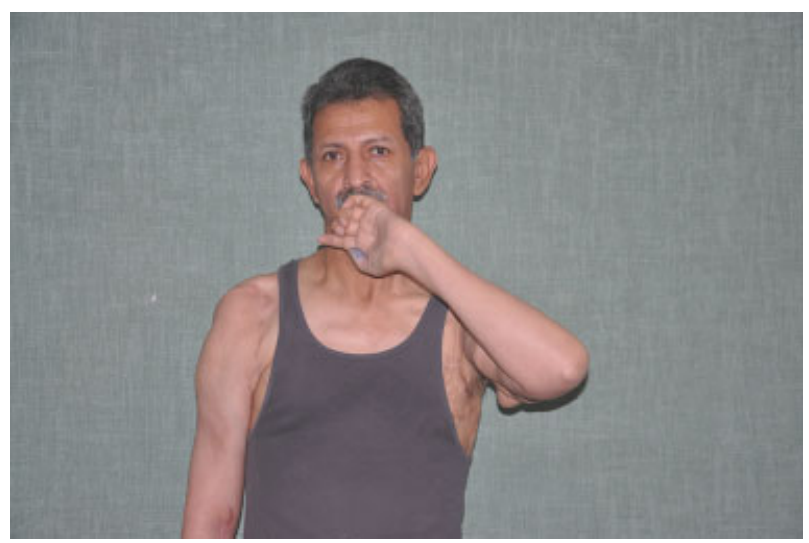

Fig. 6 Total left elbow flexion. syndrome can occur with a total ischemia time of less than 4 hours and can be fatal in cases of arm transplantation. ${ }^{27}$ To avoid this in the right arm, arteriorrhaphy was initially performed, and the venous return was allowed to drain without phleborrhaphy. Thus, we avoided the flow of any lysis products (myoglobin) and cytokines to the recipient. The disadvantage was the significant bleeding, which led to a requirement for massive transfusion with its potential risks.

The right arm was transplanted with an intact humerus, as the residual humeral head had marked osteopenia. This is consistent with previously reported amputations, in which, regardless of the mechanism of amputation, the residual bones have shown marked osteopenia due to disuse or injury. ${ }^{17}$ Additionally, fractures of the humeral neck carry the risk of inadequate consolidation, even with the application of osteosynthesis material, due to disruption of circulation of the humeral head. ${ }^{28}$ Furthermore, the mechanism of amputation in the present case was a high-voltage electric burn, which undoubtedly affected the microcirculation of the humeral head.

The recovery of intact muscles as a functional unit in both arms allowed us to reinsert them into their corresponding origins, thereby, avoiding midlevel muscle repair in the left arm, diminishing the scar, and allowing us to correctly manipulate the tendon and muscle tension. In this left arm, insertion and innervation of the muscular stumps of both the biceps and triceps brachii were preserved. These were repaired with the "piggyback" technique to increase the chances of obtaining adequate elbow flexion. These principles and technique have been previously described by Carlsen and Schnneberger. ${ }^{17,18}$

Although end-to-side neural repair is more experimental than clinical, ${ }^{29,30}$ the musculocutaneous nerve of the donor was repaired end-lateral to the recipient radial nerve in the left arm at its proximal third. This was because the biceps brachii muscle stumps, although atrophied, presented with good contraction, and we chose not to lose its function when performing end-to-end repair between musculocutaneous nerves. It was also surgically more accessible to perform this neurorrhaphy on the upper third of the radial nerve. The possibility of not obtaining reinnervation of the brachial biceps donor was contemplated. In this situation, the elbow flexion would be recovered with the function of the recipient stumps of the brachial biceps and the transference of pectoralis major muscle.

The reinnervation in the present case has been clinically evaluated for the presence of mobility. The results of electrophysiological studies will be reported later. However, we consider important to report that the motor unit action potential on the left donor biceps brachii was registered at 5 postoperative months and increased its amplitude gradually until reaching $500 \mathrm{mV}$ at 14 months posttransplant, compared with the right biceps brachii that reached $826 \mathrm{mV}$ at the same time.

Although at postoperative day 306, there was clinical and electrophysiological activity in the transplanted biceps, we decided to perform the pectoralis major transfer to avoid edema and subsequent fibrosis in the hands. 
The first movement recovered was flexion and extension of the left elbow, which is currently at M5. This movement is due to reinnervation of the transplanted biceps and triceps brachii. The second movement recovered was the abduction of the right shoulder, which is currently at M4. This movement is due to the repaired suprascapular tendon and the transplanted deltoid muscle. The flexion of the right elbow was reinforced with the transfer of the pectoralis major and is currently at M4. Left wrist extension is at M4, and this movement is due to the transplanted wrist extensor muscles. Digital flexion and pronosupination were incipient in both extremities. In addition, the patient had thermal discrimination and sensitivity to deep pressure.

These clinical results at postoperative 15 months are similar to those reported in previous arm transplantation cases. The patient will continue intensive rehabilitation to improve mobility.

\section{Conclusion}

Clinical results at postoperative 15 months are similar to those reported in previous arm transplantation cases. Basic concepts in arm transplantation include decreasing total ischemia time to prevent the flow of cytokines and lysis products from the graft to the recipient and transplantation of total muscle units. Surgical salvage procedures such as end-to-side neurorrhaphy were successfully applied in the present case. The description of the surgical technique and its relationship with the clinical results will enable better results to be obtained in future arm transplantations.

\section{Acknowledgments}

The authors would like to thank the people and institutions involved in the program: JD Francisco Chevez for his legal support; Mrs. Palmira de la Garza for her guidance and advice in public relations; the Department of Experimental Surgery, National Institute of Medical Science and Nutrition Salvador Zubiran (INCMNSZ); The Centro de Evaluación y Rehabilitación Biónica y Robótica (CEREBRO) for its technical support; the plastic surgeons, Raúl Favela, MD, Humberto Baeza, MD, Miguel de la Parra, MD, and Leonardo Bravo; Leonardo Abarca; Miriam N García-Alvarez; Raúl Granados-Martinez; Elizabeth Rodríguez; Juan Sebastian Wong; the orthopedic surgeon, Ricardo Parra-García; the physical therapy staff, Rocío Cervantes, Sarai ContrerasBarbosa, África Navarro-Lara; the surgical nurses' staff, Najera-Ortiz P, Navarrete-Romero M, Martinez-Becerril; M, Lopez Camacho M; Salazar G; Hernandez-Capultitla MC; Del Valle-Martínez S; Palma Y; Ayapantecatl Melendez G; Trejo G; Contreras A; Martínez C; organ procurement coordinators, Querevalu-Murillo W, Lopez-Jimenez JL, and Bustamante-Madrigal; social security institutions, Instituto Mexicano del Seguro Social, Instituto de Seguridad Social al Servicio de los Trabajadores del Estado; Universidad Nacional Autónoma de Mexico for borrowing surgical trainning facilities; Centro Nacional de Trasplantes; the medical students, Mario A Morán, Claudio Reyes-Montero, Ignacio
Del Carmen, Euridice I Lara, Ricardo Hernández-Gallo, and Alan Hernández-Campos; the ambulance driver, Mr. Mondragón-Gallaga M; the technician, Mr. Gomez-Vergara $\mathrm{V}$; and the charitable organizations for their financial support to the patient, The Instituto Carlos Slim de la Salud and the nonprofit organization, Trasplante y Vida I.A.P.

\section{Conflict of Interest}

None.

\section{References}

1 Jones NF, Schneeberger S. Arm transplantation: prospects and visions. Transplant Proc 2009;41(02):476-480

2 Shores JT, Higgins JP, Lee WP. Above-elbow (supracondylar) arm transplantation: clinical considerations and surgical technique. Tech Hand Up Extrem Surg 2013;17(04):221-227

3 Salminger S, Roche AD, Sturma A, Mayer JA, Aszmann OC. Hand transplantation versus hand prosthetics: pros and cons. Curr Surg Rep 2016;4:8

4 Iglesias M, Leal P, Butrón P, et al. Severe complications after bilateral upper extremity transplantation: a case report. Transplantation 2014;98(03):e16-e17

5 Messner F, Grahammer J, Hautz T, Brandacher G, Schneeberger S. Ischemia/reperfusion injury in vascularized tissue allotransplantation: tissue damage and clinical relevance. Curr Opin Organ Transplant 2016;21(05):503-509

6 Tuffs A. Munich surgeons perform world's first transplantation of whole arms. BMJ 2008;337:a1162

7 Cavadas PC, Ibáñez J, Thione A, Alfaro L. Bilateral trans-humeral arm transplantation: result at 2 years. Am J Transplant 2011; 11(05):1085-1090

8 Jablecki J, Kaczmarzyk L, Domanasiewicz A, et al. Result of armlevel upper-limb transplantation in two recipients at 19- and 30month follow-up. Ann Transplant 2012;17(03):126-132

9 Shores JT, Brandacher G, Lee WP. Hand and upper extremity transplantation: an update of outcomes in the worldwide experience. Plast Reconstr Surg 2015;135(02):351e-360e

10 Chełmoński A, Kowal K, Jabłecki J. The physical and psychosocial benefits of upper-limb transplantation: a case series of 5 polish patients. Ann Transplant 2015;20:639-648

11 Ruane ME. Johns Hopkins Hospital performs double arm transplant on Army soldier. The Washington Post. January 28, 2013. Available at: https://www.washingtonpost.com/local/johns-hopkins-hospital-performs-double-arm-transplant-on-army-soldier/2013/ 01/28/d4b8e082-673c-11e2-93e1-475791032daf_story.html? tid=a_inl\&utm_term=.01885c975b6c. Accessed December 8, 2016

12 Hand Transplant History. Composite Tissue Allotransplantation. Available at: http://www.handtransplant.com/TheProcedure/HandTransplantHistory/tabid/96/Default.aspx. Accessed December 8, 2016

13 John Peck, Bilateral Arm Transplant Patient. Brigham and Women's Hospital. Available at: http://www.brighamandwomens.org/About_BWH/publicaffairs/news/HandTransplant/ Peck.aspx. Accessed December 8, 2016

14 Schneeberger S, Landin L, Jableki J, et al; ESOT CTA Working Group. Achievements and challenges in composite tissue allotransplantation. Transpl Int 2011;24(08):760-769

15 Iglesias M, Butrón P, Moran-Romero M, et al. Bilateral forearm transplantation in Mexico: 2-year outcomes. Transplantation 2016;100(01):233-238

16 Iglesias Morales M, Butrón Gandarillas MP, García Mancilla S, et al. Descripción de muestra de receptores de extremidad superior. Rev Mex Traspl 2013;2(S1):S26. Available at: http://www.medigraphic.com/pdfs/trasplantes/rmt-2013/rmts131e.pdf 
17 Carlsen BT, Al-Mufarrej F, Moran SL. Surgical anatomy of hand allotransplantation. Clin Anat 2013;26(05):578-583

18 Schneeberger S, Ninkovic M, Gabl M, et al. First forearm transplantation: outcome at 3 years. Am J Transplant 2007;7(07):1753-1762

19 Hartzell TL, Benhaim P, Imbriglia JE, et al. Surgical and technical aspects of hand transplantation: is it just another replant? Hand Clin 2011;27(04):521-530, $\mathrm{x}$

$20 \mathrm{McDiarmid}$ SV, Azari KK. Donor-related issues in hand transplantation. Hand Clin 2011;27(04):545-552, x-xi

21 Azari KK, Imbriglia JE, Goitz RJ, et al. Technical aspects of the recipient operation in hand transplantation. J Reconstr Microsurg 2012;28(01):27-34

22 Banegas RN, Moreno R, Duggal A, Breidenbach WC III. Surgical aspects of donor hand recovery for transplantation. J Reconstr Microsurg 2012;28(01):21-26

23 Haddock NT, Chang B, Bozentka DJ, Steinberg DR, Levin LS. Technical implications in proximal forearm transplantation. Tech Hand Up Extrem Surg 2013;17(04):228-231
24 Pulos N, Shaked A, Abt PL, et al. Hand allotransplantation: operating room set-up and team coordination. Tech Hand Up Extrem Surg 2015;19(02):68-72

25 Cetrulo CL Jr, Kovach SJ. Procurement of hand and arm allografts. Tech Hand Up Extrem Surg 2013;17(04):232-238

26 Małecki R, Gacka M, Boratyńska M, et al. Assessment of vascular function of hand allografts. Ann Transplant 2014;19:621-628

27 Blaisdell FW. The pathophysiology of skeletal muscle ischemia and the reperfusion syndrome: a review. Cardiovasc Surg 2002; 10(06):620-630

28 Maier D, Jaeger M, Izadpanah K, Strohm PC, Suedkamp NP. Proximal humeral fracture treatment in adults. J Bone Joint Surg Am 2014;96(03):251-261

29 Rovak JM, Cederna PS, Macionis V, Urbanchek MS, Van Der Meulen $\mathrm{JH}$, Kuzon WM Jr. Termino-lateral neurorrhaphy: the functional axonal anatomy. Microsurgery 2000;20(01):6-14

30 Papalia I, Geuna S, D'Alcontres FS, Tos P. Origin and history of endto-side neurorrhaphy. Microsurgery 2007;27(01):56-61 